\title{
Optimization of flexible non-uniform multilevel PAM for maximizing the aggregated capacity in PON deployments
}

\section{Citation for published version (APA):}

van der Linden, R., Tran, N. C., Tangdiongga, E., \& Koonen, A. M. J. (2018). Optimization of flexible non-uniform multilevel PAM for maximizing the aggregated capacity in PON deployments. Journal of Lightwave Technology, 36(12), 2328-2336. https://doi.org/10.1109/JLT.2018.2809966

DOI:

10.1109/JLT.2018.2809966

Document status and date:

Published: 15/06/2018

\section{Document Version:}

Accepted manuscript including changes made at the peer-review stage

\section{Please check the document version of this publication:}

- A submitted manuscript is the version of the article upon submission and before peer-review. There can be important differences between the submitted version and the official published version of record. People interested in the research are advised to contact the author for the final version of the publication, or visit the $\mathrm{DOI}$ to the publisher's website.

- The final author version and the galley proof are versions of the publication after peer review.

- The final published version features the final layout of the paper including the volume, issue and page numbers.

Link to publication

\section{General rights}

Copyright and moral rights for the publications made accessible in the public portal are retained by the authors and/or other copyright owners and it is a condition of accessing publications that users recognise and abide by the legal requirements associated with these rights.

- Users may download and print one copy of any publication from the public portal for the purpose of private study or research.

- You may not further distribute the material or use it for any profit-making activity or commercial gain

- You may freely distribute the URL identifying the publication in the public portal.

If the publication is distributed under the terms of Article 25fa of the Dutch Copyright Act, indicated by the "Taverne" license above, please follow below link for the End User Agreement:

www.tue.nl/taverne

Take down policy

If you believe that this document breaches copyright please contact us at:

openaccess@tue.nl

providing details and we will investigate your claim. 


\title{
Optimization of Flexible Non-Uniform Multi-Level PAM for Maximizing the Aggregated Capacity in PON Deployments
}

\author{
Robbert van der Linden, Nguyen-Cac Tran, Eduward Tangdiongga, and Ton Koonen.
}

\begin{abstract}
Non-uniform PAM utilizes unequal distances between its modulation levels. In a multilevel PAM symbol, multiple bits are encoded. Due to the unequal level spacing some bits can be decoded successfully at a lower received optical power than others. This is well suited for practical PON deployments wherein the optical powers received by the different ONUs typically vary over a broad range. Thus, more ONUs in the PON can successfully decode non-uniform PAM-4 and PAM-8 than standard PAM-4/8, thereby increasing the aggregated capacity of the network. In systems where signal-dependent noise makes up a significant part of the total received noise level, the non-uniform PAM constellation can be adapted to take this signal-dependent variance into account. In doing so, a lower unequal level spacing can be used, decreasing the received optical power required to successfully decode all the bits in the PAM symbol. The impact of non-uniform PAM on the network throughput is presented by comparison of the experimental results with the actual loss distribution of a commercially deployed PON.
\end{abstract}

Index Terms-Flexible modulation, hierarchical modulation, passive optical networks, pulse amplitude modulation, network optimization

\section{INTRODUCTION}

D UE to the cost-sensitive nature of passive optical networks (PONs), it is important to maximize the efficiency of deployed equipment, thereby minimizing the price per bit. Efficient methods are sought to increase the data rate, while not increasing the bandwidth or sensitivity required of the transceivers [1]. By means of methods that adaptively change the deployed modulation, depending on the quality of the channel, the capacity of the network can be increased. Operating conditions within a PON are not uniform. Careful and gradual adaptation of the modulation format to the channel quality can optimize the utilization of the deployed components.

In a PON, the performance difference between links varies widely. The permitted differential optical path loss between the various Optical Network Units (ONUs) in e.g. $\mathrm{XG}(\mathrm{S})-\mathrm{PON}$ is $15 \mathrm{~dB}$ [2]. Additionally, a difference in mean optical launched power of 3 and $5 \mathrm{~dB}$ is allowed between transmitters in the down- and upstream direction, respectively. Furthermore, a difference in receiver performance can be expected, where

Manuscript submitted December 19, 2017; revised February 2, 2018; accepted February 16, 2018. Copyright (c) 2018 IEEE.

R. van der Linden (e-mail: r.v.d.linden@tue.nl) is with the Institute for Photonic Integration, Eindhoven University of Technology, Eindhoven, the Netherlands and with Genexis B.V., Eindhoven, the Netherlands

N. C. Tran is with Genexis B.V., Eindhoven, the Netherlands

E. Tangdiongga and A.M.J Koonen are with the Institute for Photonic Integration, Eindhoven University of Technology, Eindhoven, the Netherlands some receivers perform substantially better than the minimum requested performance level, while others are just operating marginally above this level. These factors combined can lead to a large margin between the actually received optical power and the minimum power required to sustain performance below the forward error correction (FEC) limit.

In previous work [3] we introduced the use of flexible modulation in PONs. Flexible modulation allocates higherorder modulation formats to those ONUs that receive enough optical power to support these formats, and at the same time allocates lower-order modulation formats to the other ONUs. More specifically, we used 4- and 8-level pulse amplitude modulation (PAM). This offers the network operator various benefits, such as doubling or tripling the data rate for the better-positioned multilevel-PAM-capable ONUs, while maintaining the standard line rate for those ONUs that only support binary on-off-keying (OOK, equivalent to PAM-2). The network operator may then decide to redistribute the timeslot distributions among the ONUs, such that they equally share the resulting increase of aggregated capacity. Or alternatively, premium services can be delivered to selected (business) users requiring a higher data rate than the other users of the network. Nevertheless, this flexible modulation scheme still does not utilize the available network capacity to its full extent. The modulation formats considered, being PAM-4 and PAM- 8 with uniformly spaced modulation levels, require at least an additional 4.8 , and $8.5 \mathrm{~dB}$ extra received power, respectively, relative to OOK to decode at the same BER. Thus, only links that receive at least $4.8 \mathrm{~dB}$ of additional optical power are able to benefit from the proposed flexible modulation scheme, which is a relatively high threshold to reap the benefits of multi-level PAM. In this work, we utilize non-uniform PAM constellations to transmit data through a constellation with unequal Euclidean distances between the constellation levels. In a multilevel PAM symbol, multiple bits are encoded. Due to the unequal level distances, some bits can be decoded with a lower BER than others. Or, equivalently, some bits can be decoded successfully below the FEC limit with a lower received optical power than others. This is well suited for practical PON deployments where different ONUs typically receive widely differing optical power levels.

The principle of non-uniform PAM can be traced back to work of Cover in 1972, based on broadcast systems [4]. In his work every user is guaranteed a basic form of communication, whereas certain users in a better situation, e.g. having less distortion in the channel, can benefit from receiving additional 


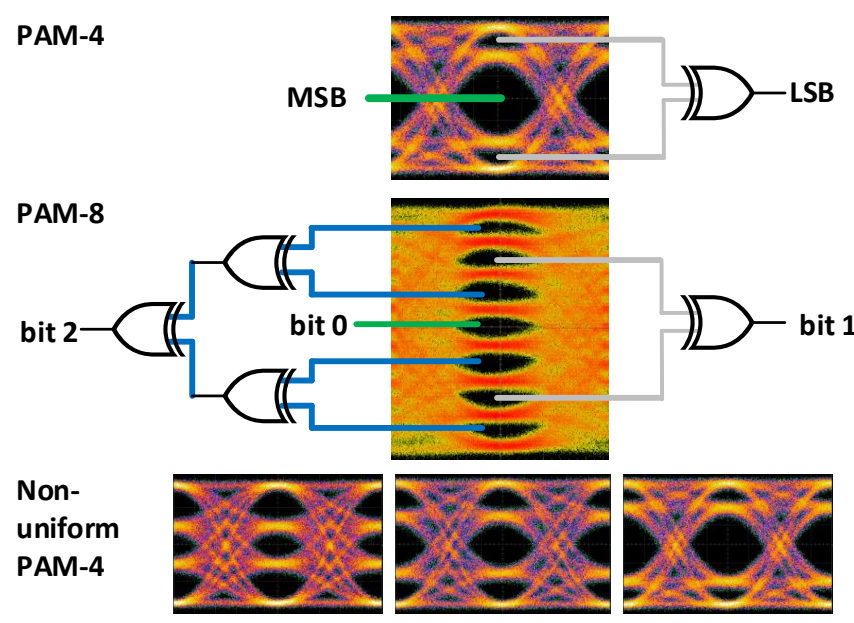

Fig. 1. Multilevel interleaving with decoding of PAM-4 (top) and PAM-8 (middle). Furthermore, non-uniform PAM-4 eye diagrams with three levels of compression (bottom). At higher compression levels the inner constellation levels move more towards the outer constellation levels.

information. Therefore, a low resolution and a high resolution video stream can be superimposed on each other [5], resulting in a more efficient broadcast transmission than time division multiplexing of low and high complexity constellations. A similar technique, named hierarchical modulation, has also been adopted in the DVB-T standard for terrestrial transmission of video signals [6].

In previous work we have shown the principle of nonuniform PAM-4 [7]. Operation in a 12.5 GBaud system has been shown in [8]. In this work, we elaborate on the proposed principle of using non-uniform PAM in a PON and validate it with experimental results of 10 Gbaud non-uniform PAM-4/8 using an APD (avalanche photodiode)-based receiver. Next, we also show the influence of signal-dependent noise on the non-uniform constellation in a 10 Gbaud SOA (semiconductor optical amplifier)-PIN photodiode based system. We experimentally verify the benefits of using a non-uniform PAM constellation optimized for signal-dependent noise inside such system. Furthermore, we show the throughput results of nonuniform PAM expected in a commercially deployed PON with an updated statistics set, encompassing a larger number of deployed ONUs than presented in previous work [3].

\section{NON-UNIFORM PAM}

In downstream PONs, the use of multilevel PAM allows multilevel interleaving [9]. With multilevel interleaving the multiple bits per PAM symbol are assigned to different users. E.g., for interleaved PAM-4, the receiver decodes the most significant bit (MSB) with a slicer having its threshold in the middle eye. The least significant bit (LSB) can be decoded by two slicers having their thresholds in the upper and lower eye, respectively, and a subsequent XOR operation. The same principle can be extended towards higher modulation orders, by having a cascade of logical gates, as shown in Fig. 1 for PAM-4 and PAM-8. Multilevel interleaving can simplify the electronics of the receiver by reducing the data rate to be processed, while still providing the same aggregated capacity of the PON as a normal PAM scheme would do.
The concept of flexible modulation, i.e. using a different order of modulation for different users within the same network depending on their received signal quality, and multilevel interleaving of ONUs can be combined to the concept of non-uniform PAM. Non-uniform PAM has unequally spaced modulation levels. E.g., in non-uniform PAM-4 the distance between the inner two levels is larger than the distance from the outer two levels to the inner ones. Therefore, the MSB of a received symbol can be successfully decoded at a lower received optical power at the expense of higher received power needed to decode the LSB. This is advantageous in a PON that utilizes flexible modulation and due to its layout has a large spread in the received powers by ONUs connected to a particular OLT port. By means of non-uniform PAM, an ONU that does not receive enough optical power to decode a standard PAM symbol, can successfully decode the MSB of a non-uniform PAM-4 symbol. This is achieved, by multilevel interleaving it with other ONUs, connected to the same OLT port, that have a surplus in received optical power. While it does not increase the peak data rate of that ONU, it does enable simultaneous transmission of two data streams, thereby it increases the aggregated data rate and decreases the congestion probability of the PON. Non-uniform PAM-4 is generated at the transmitter by generating a constellation level diagram $T$ in which a compression factor $\beta$ is introduced, by

$$
T=[-3,-1-(2 \beta),+1+(2 \beta),+3],
$$

with $0 \leq \beta<1$.

Similarly, non-uniform PAM can be utilized in PAM-8. In this case, three bits are available in a symbol (annotated by bit 0,1 , and 2), which can be transmitted to up to three different ONUs. In non-uniform PAM-4 allocation of more constellation space for one bit automatically means a reduction in available constellation space for the second bit. Due to the three bits in non-uniform PAM-8, more intermediate forms are available. In this work we explore three options. For each option bit 0 is allocated the largest part of the constellation space, and bit 2 is allocated the smallest part. The constellation space for bit 1 is set to follow either bit 0 , bit 2 or to be kept constant. The transmitted constellation for PAM- 8 with minimum bit 1 space is defined as

$$
\begin{aligned}
& T=[-7,-5-2 \beta,-3-4 \beta,-1-6 \beta, \\
& +1+6 \beta,+3+4 \beta,+5+2 \beta,+7] .
\end{aligned}
$$

The constellation of PAM- 8 with constant bit 1 space is

$$
\begin{aligned}
T=[-7, & -\frac{5+9 \beta}{1+\beta},-\frac{3+7 \beta}{1+\beta},-\frac{1+9 \beta}{1+\beta}, \\
& \left.+\frac{1+9 \beta}{1+\beta},+\frac{3+7 \beta}{1+\beta},+\frac{5+9 \beta}{1+\beta},+7\right] .
\end{aligned}
$$

And the constellation for PAM- 8 with maximum bit 1 space is defined as

$$
\begin{aligned}
T=\left[-7,-\frac{5+\beta}{1-\beta / 7},-\frac{3-\beta}{1-\beta / 7},-\frac{1+\beta}{1-\beta / 7},\right. \\
\left.+\frac{1+\beta}{1-\beta / 7},+\frac{3-\beta}{1-\beta / 7},+\frac{5+\beta}{1-\beta / 7},+7\right] .
\end{aligned}
$$




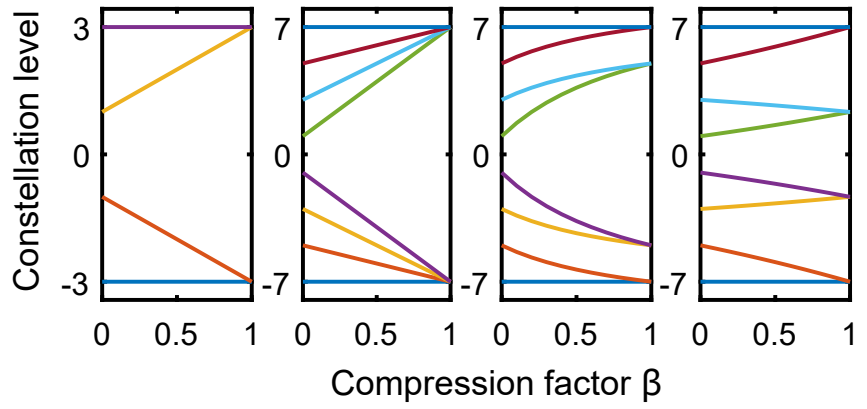

Fig. 2. Constellations of non-uniform PAM as a function of compression factor $\beta$. From left to right: PAM-4 based on (1), PAM-8 with minimum bit 1 space based on (2), PAM- 8 with constant bit 1 space based on (3), PAM-8 with maximum bit 1 space based on (4).

The relative position of the transmitted constellation levels (1)(4) are visualized in Fig. 2.

At the ONU side of the network, the receiver has to be able to adapt the threshold levels of the slicers. Decoding of bit 0 can easily be done with a slicer at a threshold level in the middle of the received symbol, irrespective of the used compression factor. The slicer threshold for decoding of the bit 1 and 2 is dependent on the compression factor.

On the medium access control (MAC) layer, algorithms have to be implemented to optimize the pairing process of ONUs for multiplexed transmission. These algorithms would have to pair low received power ONUs with high received power ONUs, thereby targeting the maximum amount of possible pairs, while not letting the average BER increase unnecessary. A simple implementation would be to start forming pairs by the lowest received power ONU, with the next higher received power ONU for any applicable $\beta$. The increase in aggregated data rate is dependent on the distribution of ONUs that request data to be transmitted. The increase in aggregated data rate is optimal if the OLT can combine packets to all ONUs. In practice, buffer length in the OLT needs to be limited, so a trade-off would have to be made. In this paper we assume an equal load distribution among the ONUs, and thus that all ONUs connected to an OLT port are requesting transmission.

An alternative concept to adapt the used data rate to the channel quality would be by the use of adaptive FEC [10]. A more complex FEC, with larger computational complexity and overhead can be used for channels with a lower SNR. In this case the gross data rate stays the same, but the net data rate available for the users decreases if the more complex FEC is enabled. A downside of this approach would be that each ONU would have to be able to perform these more computational intensive operations, regardless if the FEC is enabled or not.

Upstream communication is not considered, as non-uniform PAM is intended purely for downstream applications. Upstream transmission would be complicated by the reception of signals from two different ONUs, with two different lasers, at a single receiver. Upstream non-uniform PAM will require synchronization of the received signals at a sub-bit time scale, which is non-trivial to realize. Additionally, if the multiple transmitters generate nominally the same wavelength, the resulting beat-noise will impair the communication. When

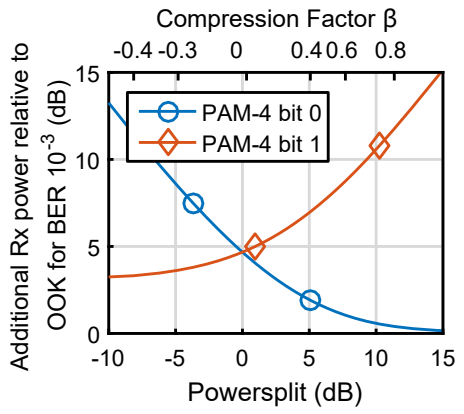

(a)

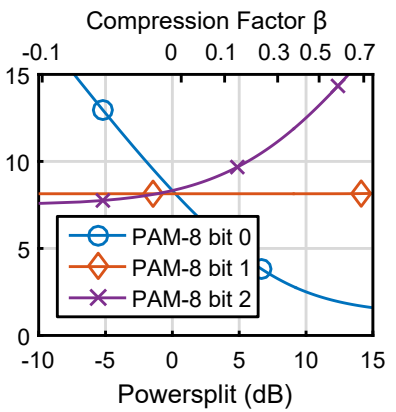

(b)
Fig. 3. Analytical results for non-uniform PAM-4/8. Additional received power relative to OOK that is required for BER $10^{-3}$ as a function of power split. Power split is defined as the spread in received powers between bit 0 and the highest bit in the symbol. The relation of power split to the compression factor $\beta$ is visualized by the top x-axis. (a): PAM-4 (b): PAM-8 with constant constellation space for bit 1 . The markers are only for visual guiding, and do not represent a specific data point.

separated wavelengths are used, the system becomes a WDM system, and the use of a WDM filter will be more practical than the use of non-uniform PAM.

\section{NUMERICAL ANALYSIS}

Fig. 3 shows the theoretical possibilities of non-uniform PAM. The figure shows an analytical calculation of the additional optical power relative to OOK modulation that is required to decode a bit from the non-uniform PAM-4 symbol successfully at the BER $10^{-3}$ level. Power split is defined as the difference in received optical powers required for bit 0 at the BER $10^{-3}$ level and the received optical power required for the highest bit in the symbol at that same BER level, a positive power split is thus achieved by positive values of $\beta$, as defined in (1)-(4). In this analysis only thermal noise is considered, signal dependent noise and intersymbol interference effects are neglected. Under these assumptions, it can be observed in Fig. 3a that for PAM-4, the required power to decode the MSB can be brought asymptotically close to the power required to decode OOK, simultaneously the power required to decode the LSB increases accordingly. In Fig. 3b, PAM-8 with constant space for bit 1 is visualized. It can be noted that the same principle of non-uniform PAM can also be applied for negative $\beta$. However, as the figure shows for negative power splits, the results are much less favourable. As the higher bits are decoded by more slicers, a larger increase in the allocated constellation space has to be assigned to these higher bits to reduce the BER, compared to the lower bits.

Furthermore, it is emphasized that, under these assumptions, the average BER for the various bits combined is at its minimum value when the constellation is equidistant or uniform. The use of non-uniform PAM is thus only beneficial if a PON is present where a spread in received optical powers is observed.

\section{APD BASED MEASUREMENT: EXPERIMENTAL SETUP AND RESULTS}

The downstream experimental setup is shown in Fig. 4. A $7.5 \mathrm{GHz}$ arbitrary waveform generator is used to generate 


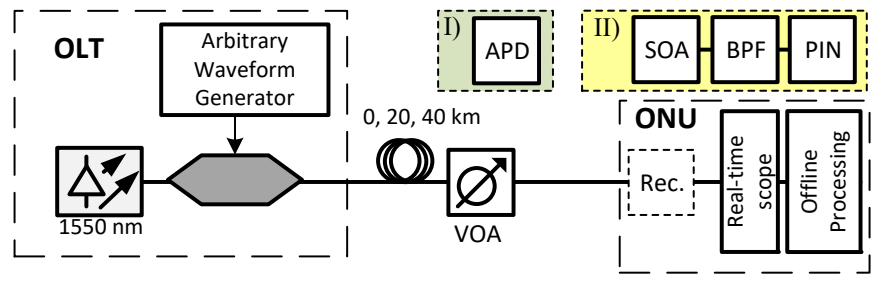

Fig. 4. The experimental setup. The receiver in the ONU consists either of I) an APD, or II) an SOA-PIN receiver with optical band pass filter. VOA: Variable Optical Attenuator.

10 Gbaud PAM signals with a constellation as given by (1)(4) for various $\beta$. The maximum supported sample rate of the AWG is $12 \mathrm{GSa} / \mathrm{s}$, during the experiment the AWG generates $10 \mathrm{GSa} / \mathrm{s}$ without oversampling of the PAM symbols. The PAM signal is used to modulate light from a $1550 \mathrm{~nm} \mathrm{CW}$ DFB laser with a Mach-Zehnder modulator (MZM). After optional transmission over 20 , or $40 \mathrm{~km}$ of fiber, the signal is received by a $10 \mathrm{Gbps}$ rated APD. This signal is captured by a real-time oscilloscope and stored for offline processing, in which no advanced techniques are utilized. The various bits in the multilevel symbol are decoded separately, the location of the slicers thresholds is halfway between two transmitted constellation levels. Optionally, if an equalizer is used, the signal is equalized by a least mean squares feedforward equalizer consisting of 31 symbol spaced taps.

It should be noted that although we used a DAC to generate the signals, this is not strictly required. Two 1-bit pulse generators with an adjustable output voltage, combined with a power splitter, are sufficient to generate any standard non-uniform PAM-4 constellation. Similarly, three 1-bit pulse generators can generate the non-uniform PAM- 8 constellation.

Fig. 5 depicts the experimental BER results by showing the required received optical power to decode each of the bits contained in the multilevel PAM-4/8 symbol at a BER level of $10^{-3}$, as a function of the non-uniformity of the transmitted constellation diagram. With a constant symbol rate of 10 Gbaud, the aggregated bit rate of PAM-4/8 is 20/30 Gbps. It can be seen that the experiment agrees with the analytical simulation shown in Fig. 3. Performance of nonuniform PAM-4 is shown in Fig. 5a. For visual clarity, Fig. 5b shows a close-up of Fig. 5a for small $\beta$. The power required to decode both PAM-4 bits in an equidistant constellation with an average BER below $10^{-3}$ is $-23.1 \mathrm{dBm}$ without an equalizer, and $-24.1 \mathrm{dBm}$ with the 31-tap FFE, thereby, requiring 6.7 and $5.6 \mathrm{~dB}$ more optical power than decoding of $10 \mathrm{Gbps}$ OOK signals requires. The influence of the equalizer on the performance of the MSB is negligible, however the influence is much more pronounced for the performance of the LSB for higher compression factors. Whereas the FFE improves the performance of equidistant PAM-4 with $1.0 \mathrm{~dB}$, the benefit in a non-uniform system can be larger. E.g. in a PON where an ONU with $-15 \mathrm{dBm}$ received optical power is available for the LSB, non-uniform PAM-4 w/o EQ can decode the MSB at $-26.9 \mathrm{dBm}$. If the FFE is utilized, a higher compression factor can be used, due to the better performance of the LSB with the FFE, and therefore the MSB can instead be decoded at -
$29.0 \mathrm{dBm}$, an improvement of $2.1 \mathrm{~dB}$ compared to the system w/o FFE. A similar trend is visible for the influence of the fiber transmission distance. It is negligible for the MSB of PAM-4, the LSB does show a slight influence. As the data was modulated with an MZM, we would expect to see a more pronounced influence of dispersion if the signal would be transmitted with a DML or EML.

Performance of the three versions of non-uniform PAM-8, with the middle bit allocated the minimum, constant, or maximum part of the constellation space is shown in Fig. 5c, $5 d$, and $5 f$ respectively. Decoding of all three bits of PAM-8 at an average BER level of $10^{-3}$ with the FFE requires $-17.9 \mathrm{dBm}$, $11.9 \mathrm{~dB}$ more than OOK. Decoding of PAM-8 without an equalizer at a BER level below $10^{-3}$ is not feasible due to the appearance of an error floor above this level, regardless if a non-uniform constellation is used or not. Fig. 5c shows the influence of the fiber distance on the BER results, similar to PAM-4, the fiber distance mainly shows in bit 2 . For visual clarity, Fig. 5e shows a close-up of Fig. $5 \mathrm{~d}$ for small $\beta$.

We also measured a time interleaved transmission, where after each block of 128 symbols the compression factor was changed. We observed no penalty due to this time-interleaved transmission, relative to continuous transmission with the same compression factor.

\section{Network PERformance}

To examine the effect that non-uniform PAM can have on the aggregated capacity of a PON we cross-reference the experimental results with a data set of received optical powers of more than 45,000 ONUs from the GPON networks of INEA, Poland. This data set is an extension of the data set used in previous works [3], consisting of partly the same ONUs as the previous data set. The ONUs of this network use B+ class optics. The percentage of ONUs that can operate with a certain modulation format is estimated by calculating the experimentally obtained power penalty between OOK and this modulation format. This penalty is then added to the OOK receiver sensitivity as defined in the $\mathrm{B}+$ class $(-27 \mathrm{dBm})$, and the percentage of ONUs with more power than this number is calculated with the loss statistics of the PON. Although this method has its limitations, it is preferred over a direct comparison of the experimentally obtained results. This would give us an unfair advantage, as the OOK performance of our system is several $\mathrm{dB}$ better than what is guaranteed in the various PON standards. The goal of this work is not to show the influence of a better receiver, but to show the influence of non-uniform PAM, given a certain receiver performance.

The achievable increase in aggregated data rate depends on the distribution of received optical powers in the network, especially the spread per OLT port. Based on the experimental results in this paper, obtained with an APD receiver, and the loss distribution of the deployed network, 64\% of the ONUs would be capable of using equidistant PAM-4 without equalization, and $77 \%$ with the FFE. Support for PAM-8 is limited to $5 \%$ of the ONUs. Therefore the aggregated capacity of a 10G-class network with flexible modulation, without non-uniform constellations, is expected to be 16.4, 17.7, or 


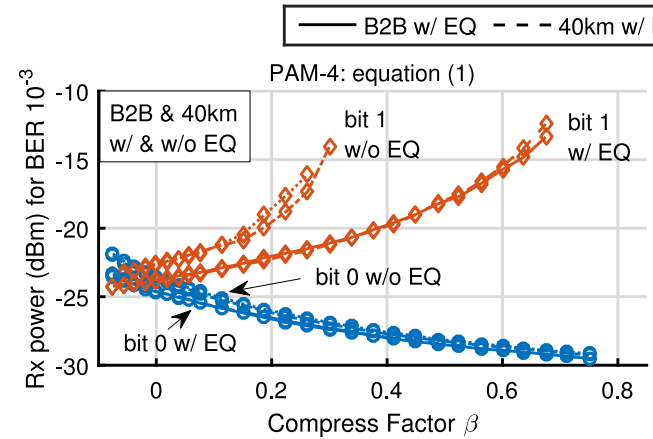

(a)

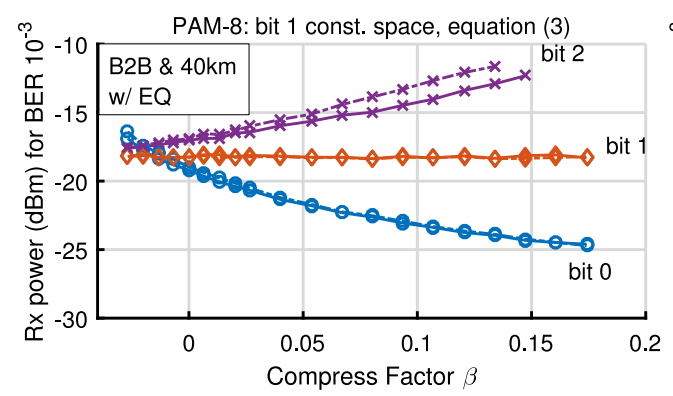

(d)

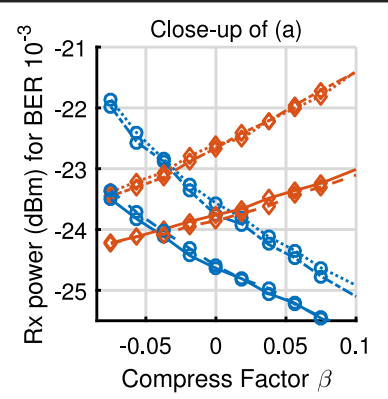

(b)

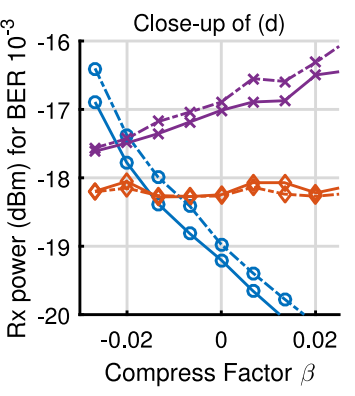

(e)

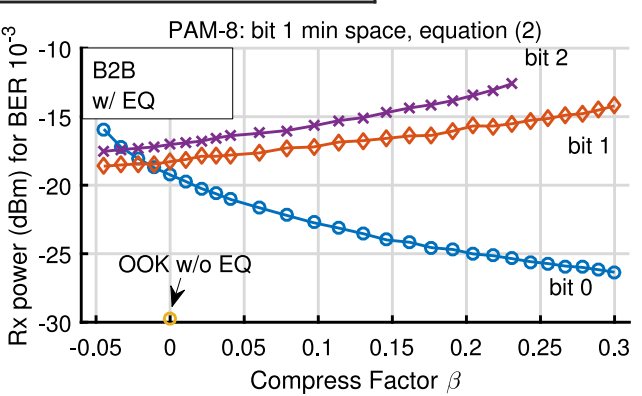

(c)

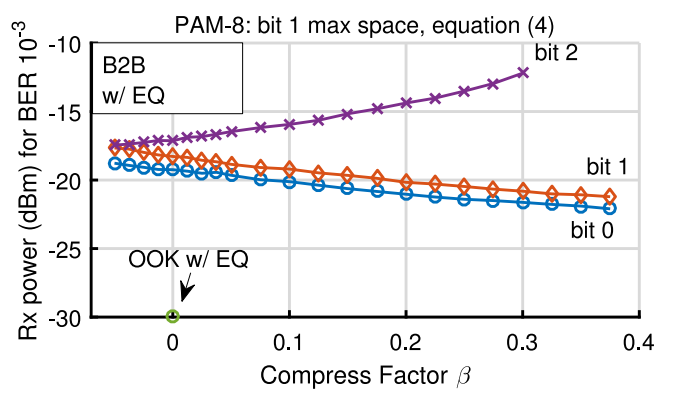

(f)

Fig. 5. Experimental results showing the required received optical power at the APD receiver to decode a bit at a BER level of $10^{-3}$. The symbol rate is constant at 10 Gbaud. (a): PAM-4 w/ and w/o EQ both B2B and over $40 \mathrm{~km}$. (b): Close-up of the same results in (a) for small $\beta$ (c): PAM-8 with minimal constellation space for bit 1, B2B (d): PAM-8 with constant constellation space for bit 1, B2B \& $40 \mathrm{~km}$ (e): Close-up of the same results as in (d) for small $\beta$. (f): PAM-8 with maximum constellation space for bit $1, \mathrm{~B} 2 \mathrm{~B}$ only

$18.3 \mathrm{Gbps}$, for a system using flexible PAM-4 alone w/o EQ, $\mathrm{w} / \mathrm{EQ}$, and in a combination of PAM-4 w/ EQ and PAM-8 $\mathrm{w} / \mathrm{EQ}$, respectively. The distribution of downstream received optical powers and the support for equidistant PAM-4 and PAM-8 w/ EQ is shown in Fig. 6a.

With non-uniform PAM-4 w/o FFE, 75\% of the ONUs is capable of receiving PAM-4. With FFE this increases to $87 \%$. Non-uniform PAM- 8 can be received by $7 \%$ of the ONUs. Thus the aggregated capacity of a 10G-class network with non-uniform constellations would expected to be 17.5, 18.7, and 19.4 Gbps for a system using flexible non-uniform PAM-4 alone w/o EQ, w/ EQ, and in a combination of non-uniform PAM-4 w/ EQ and PAM-8 w/ EQ, respectively. The ONUs able to receive non-uniform PAM-4/8 w/ EQ are shown in Fig. 6b. This assumes an equal capacity demand by all users.

As such it can be observed that the majority of the capacity increase can be achieved by the use of PAM-4, in either an equidistant or a non-uniform form. In the examined network, the additional improvement in capacity by using of PAM-8 is substantially smaller due to the large additional required received optical power. A substantial influence of using nonuniform PAM would be expected for networks with an increased spread in received optical powers per OLT port.

The results presented here are for PAM- 8 with bit 1 allocated the maximum constellation space, percentages of ONUs that can use non-uniform PAM- 8 where bit 1 has the minimum or constant constellation space are virtually identical.

\section{SIGNAL-DEPENDENT NOISE OPTIMIZATION}

In scenarios where a larger power budget is required than that is feasible with an APD-based receiver, a SOA-PIN based receiver can be used. A SOA-PIN receiver combined with an optical bandpass filter typically shows better performance than an APD based receiver [11]. However, the noise variance $\sigma^{2}$ in a PON with a SOA-PIN based receiver shows stronger signaldependent characteristics than in systems utilizing APD or PIN receivers [12]. In an unamplified PIN based receiver, thermal noise is dominant, which is independent of the received power level. Inside an APD the received signal is amplified, due to the avalanche process the resulting signal has a higher shot noise contribution. In APD based systems shot noise can be dominant, depending on this excess noise factor of the APD. In an SOA-PIN based receiver the noise variance has a linear dependency on the received optical power due to signal-ASE beating noise and shot noise. All systems show a quadratic noise variance dependency on the received power due to relative intensity noise of the transmitting laser and due to distortions on the transmitting signal. In practice both an APD and a SOA-PIN based receiver exhibit signal-dependent noise, however the relative contribution compared to the signalindependent thermal noise is larger for the SOA-PIN receiver.

In a signal-dependent noise dominated transmission, the BER will be dominated by the errors on the upper constellation levels. This is true for every transmission, but has a more pronounced effect in non-uniform PAM transmissions. Therefore, in the next section we show the influence of the 


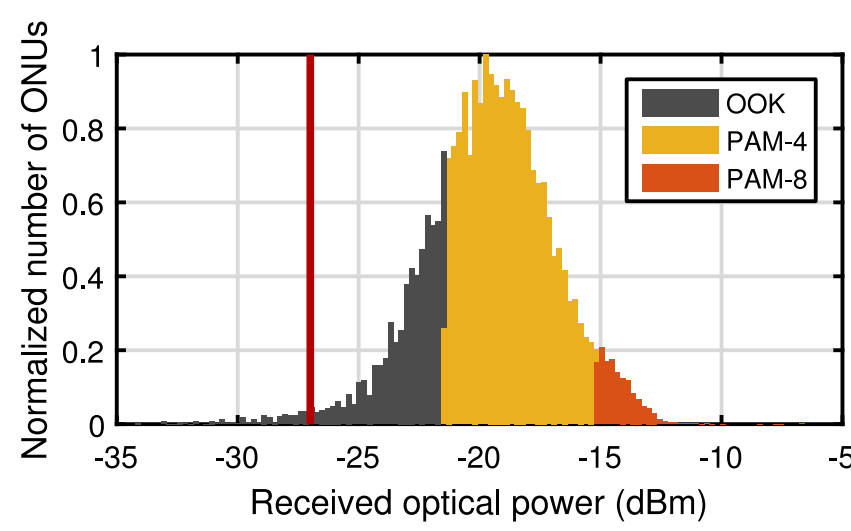

(a)

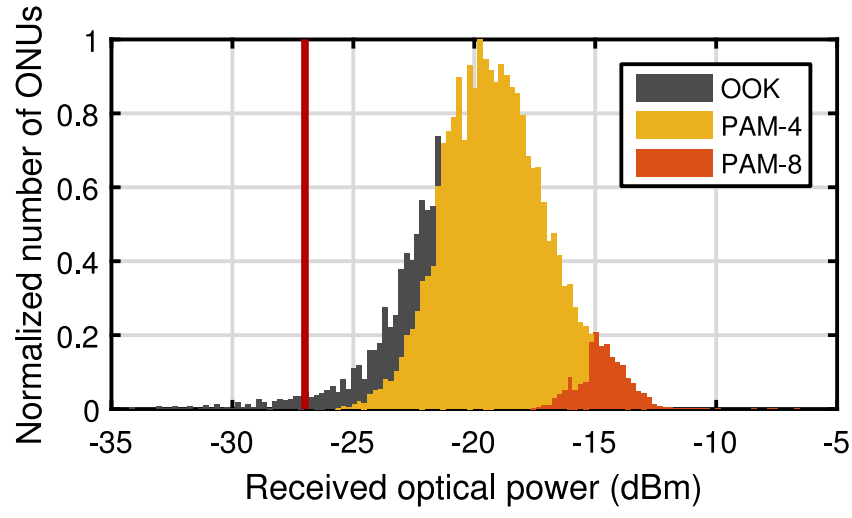

(b)

Fig. 6. Normalized histogram of received optical downstream powers, measured at 45,000 ONUs in the networks of INEA. The OOK sensitivity for a B+ ONU receiver is shown by the vertical red line. (a): Shows the fraction of ONUs that are able to decode equidistant PAM-4/8 w/ EQ without non-uniform PAM. (b): Shows the fraction of ONUs that can decode non-uniform PAM-4/8 w/ EQ successfully below the FEC limit.

signal-dependent noise on a non-uniform PAM transmission, and show the construction of a non-uniform PAM constellation optimized for the presence of signal-dependent noise. We do so using an SOA-PIN based receiver.

If the noise power around each constellation level is known, the transmitted constellation can be adapted accordingly by allocating a relative larger part of the constellation space for these upper constellation levels [13]. It is well known that, under Gaussian approximation, the error probability at threshold $k$ is

$$
P_{e}(k)=\frac{1}{2} \operatorname{erfc}\left(\frac{Q_{k}}{\sqrt{2}}\right),
$$

where $\operatorname{erfc}(x)$ is the complementary error function and $Q_{k}$ is the $\mathrm{Q}$ factor at threshold $k$, defined as

$$
Q_{k}=\frac{I_{k+1}-I_{k}}{\sigma_{k+1}+\sigma_{k}},
$$

where $I_{k}$ is the received signal level at the $k$ th constellation level, and $\sigma_{k}$ is the root mean square value of the noise at the $k$ th constellation level. Therefore, if the desired $Q_{k}$ are known, it is possible to calculate the required received signal levels to achieve a certain target BER by iteratively evaluating

$$
I_{k}^{i}=I_{k-1}^{i}+Q_{k}\left[\sigma_{k-1}\left(I_{k-1}^{i}\right)+\sigma_{k}\left(I_{k}^{i-1}\right)\right]
$$

over iterations $i$. The final result gives the constellation shape that should be transmitted, and the received optical power level that is required for decoding.

Our target application is a PON with a broad range of received optical powers, where the multiple bits in a single PAM symbol are decoded simultaneously at different power levels. As such, (7) has to be extended to take into account these multiple power levels, and corresponding signaldependent noise levels. Furthermore, in our experimental work we observed a dependency of the noise power on both the mean received optical power and the received optical power of a specific constellation level. Therefore, in the subsequent experimental work we do not use analytical expressions [12] to approximate the noise power for each constellation level,

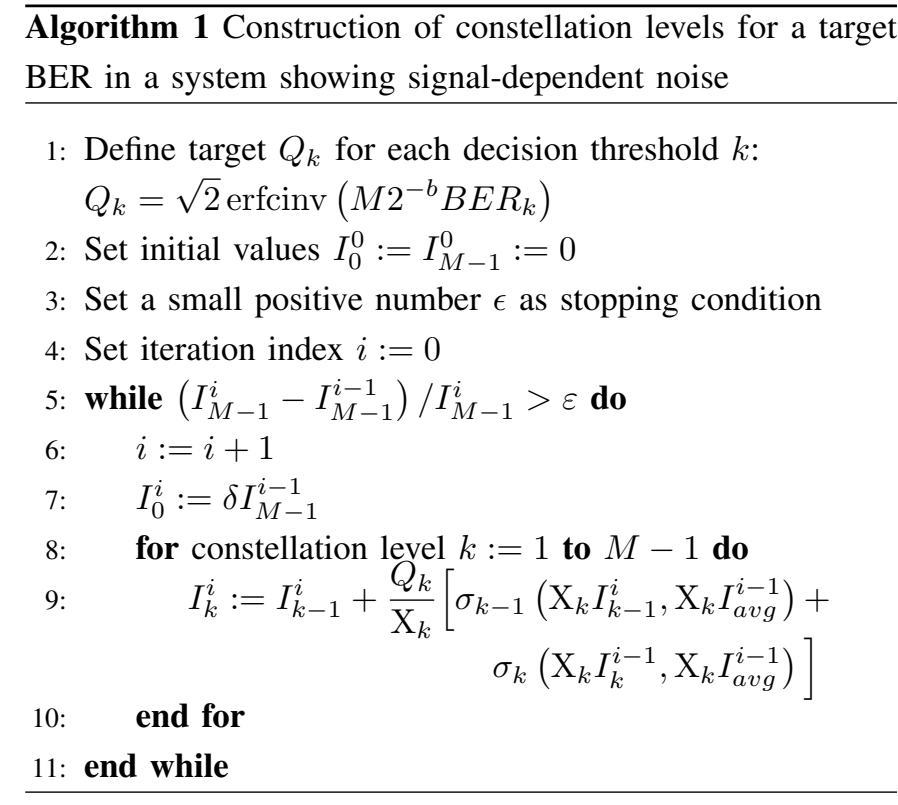

but use a two dimensional quadratic fit of the measured $\sigma^{2}$ to form an expression of $\sigma^{2}$ as a function of the optical power level of the received constellation level, as well as to mean received optical power.

The principle of the construction of the constellation is to merge multiple sub-constellations that are optimized for a certain power level. The key is that each decision threshold is only important at one single power level. A PAM symbol represents several bits $b$. First the average received optical power levels $\chi_{b}$ at which the constellation needs to be optimal for the various bits are defined. That is, the MSB of PAM-4 is being evaluated at power level $\chi_{0}$, the LSB is considered at power level $\chi_{1}$. Further an array $X$ is defined, that for each decision threshold defines the ratio of the received power between the bit that is being decoded at this threshold relative to the power received at the MSB decoding location. The array 
$\mathrm{X}$ for PAM-4 is defined as

$$
\mathrm{X}=\left[\begin{array}{lll}
\frac{\chi_{1}}{\chi_{0}} & 1 & \frac{\chi_{1}}{\chi_{0}}
\end{array}\right]
$$

Subsequently, the actions as represented in Algorithm 1 are performed, an extension to [13]. Here, $\delta$ is the extinction ratio of the modulator used, such that $\delta=I_{0} / I_{M-1}$. This algorithm creates the constellation diagram guaranteeing a BER corresponding to $Q_{k}$ for a given split in received powers. Typically, less than 6 iterations are required to achieve an equilibrium constellation with an error $\epsilon<1 \%$. In practice, the OLT could create a look up table with results of this algorithm. When communication to an ONU pair is required, the look up table can be accessed wether communication is possible at the respective ONU powers, and if so which constellation will give a BER of at least the value belonging to the $Q_{k}$ 's.

To alleviate the calculation requirements on the OLT, this LUT can alternatively be calculated in advance and not during runtime. In this case, actual performance may not be optimal, as e.g., differences in performance characteristics among specific transceivers cannot be taken into account.

The maximum achievable $Q_{k}$ is not known a priori. Therefore, the target $Q_{k}$ is to be set such that the BER is below the FEC limit of the system. The constructed constellation will be optimal at the FEC limit, and will have a lower BER if more power is received. If the system is to be optimized any further, a new iteration with higher $Q_{k}$ can provide a further reduction of the BER.

Equations (1)-(4) should not be used to describe the constructed constellation, instead we will refer to the constructed constellations by their resulting power split between the lowest and highest bit.

\section{SOA-PIN BASED MEASUREMENT: EXPERIMENTAL SETUP AND RESULTS}

The experimental verification of the influence of the signaldependent noise non-uniform PAM constellation is performed on a setup similar to the setup for the APD based measurement, and is shown in Fig. 4. First, the link is characterized by measuring the noise variance over a range of mean optical input powers and instantaneous powers of a specific constellation level. The noise variance measurement transmits a standard non-uniform PAM-4 signal. Consecutive PAM-4 constellations with different compression factors $\beta$ are transmitted to cover the range of possible received constellation levels. This process is repeated for the different mean received optical powers by changing the variable optical attenuator in the setup, which simulates the different mean powers received by the different ONUs. The variance $\sigma^{2}$ is obtained by measuring the variance in signal levels just before decoding. To minimize the residual intersymbol interference, and therefore the over-approximation of the noise variance, the same equalizer is used as is in the APD setup. Next, a two-dimensional quadratic fit is made on the measured $\sigma^{2}$ as a function of mean input power to the SOA-PIN and the received signal level for a constellation level. The resulting function for $\sigma^{2}$ is used to construct the constellation through algorithm 1. In the constructed constellation, the probability of error is equal at each threshold level, and thereby the required optical power is minimized. After creation of the signal-dependent noise optimized non-uniform PAM constellation, the BER measurement is performed identical to the experiment with the APD-based receiver.

The experimental results are shown in Fig. 7. This figure shows the required received optical power to decode a bit at a BER level of $10^{-3}$ for an SOA-PIN based receiver, for a standard non-uniform PAM constellation and a signaldependent noise optimized non-uniform PAM constellation, as a function of the difference between the required powers for the MSB and LSB. Fig. 7a shows the results without the use of the FFE. Although it should be noted, that the noise-dependent constellation itself was constructed beforehand with the help of the FFE. Fig. $7 \mathrm{~b}$ shows the results with the FFE enabled. In this SOA-PIN based measurement the power required to decode both the MSB and LSB of an equidistant PAM-4 constellation with an average BER below the $10^{-3}$ level is -27.3 , and $-27.7 \mathrm{dBm}$ with and without the FFE, respectively. Thereby equidistant PAM-4 has a penalty compared to OOK decoding of 7.3 and $6.9 \mathrm{~dB}$, respectively. With the signaldependent noise optimized constellation, $-28.0 \mathrm{dBm}$ (w/o $\mathrm{FFE}$ ), and $-28.5 \mathrm{dBm}(\mathrm{w} / \mathrm{FFE})$ is required, thus reducing the penalty relative to $\mathrm{OOK}$ to 6.7 and $6.1 \mathrm{~dB}$, respectively.

The influence of the noise optimization can be larger for a non-uniform PAM transmission, than for a standard transmission as the penalty for LSB decoding for high compression factors increases asymptotically. E.g. in a SOA-PIN w/ FFE based system without noise dependent constellation where one ONU only has $-32 \mathrm{dBm}$ power available (Point A in Fig. 7b), it would have to be paired together with another ONU that receives $-22.8 \mathrm{dBm}$ optical power (point $\mathrm{B}$ ) to enable both ONUs to successfully decode their respective bits. In the noise optimized constellation, a smaller amount of compression can be applied (point $\mathrm{C}$ ), due to the better base sensitivity of this constellation. Therefore the LSB ONU only requires $25.6 \mathrm{dBm}$ (point D), a decrease of $2.8 \mathrm{~dB}$. The difference in required power between the standard and noise optimized constellations depends on the amount of compression that is required.

\section{SOA-PIN NETwORK PERFORMANCE}

Based on the experimental results with the SOA-PIN receiver, Table I presents the percentage of ONUs in the deployed INEA network that would be able to decode the various formats of PAM-4. As can be seen, adapting the transmitted constellation to take into account the signal-dependent noise levels, increases the percentage of ONUs that would be able to decode non-unform PAM-4 by $11 \%$, to $82 \%$. Thus, in a 10 Gbps rated PON network equipped with SOA-PIN receivers, the aggregated capacity would be expected to increase to 18.2 Gbps.

The SOA-PIN has a lower increase in aggregated capacity than the APD receiver. The added benefit of flexible modulation and non-uniform PAM to a deployed network depends on the power penalty of the higher order PAM compared to 10 Gbps OOK with that same receiver, not on the absolute receiver performance. The SOA-PIN has an improved absolute sensitivity for both OOK and PAM-4, but has a larger 


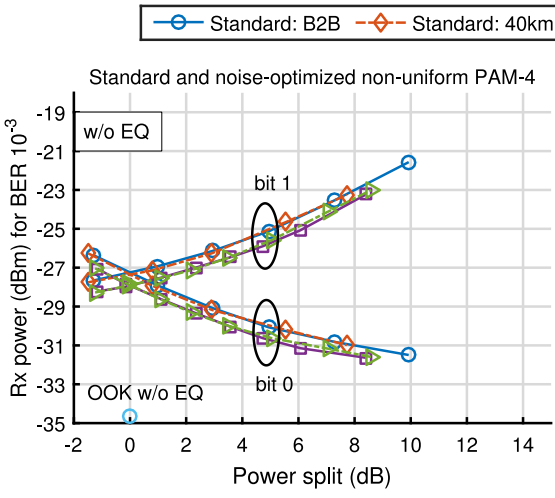

(a)

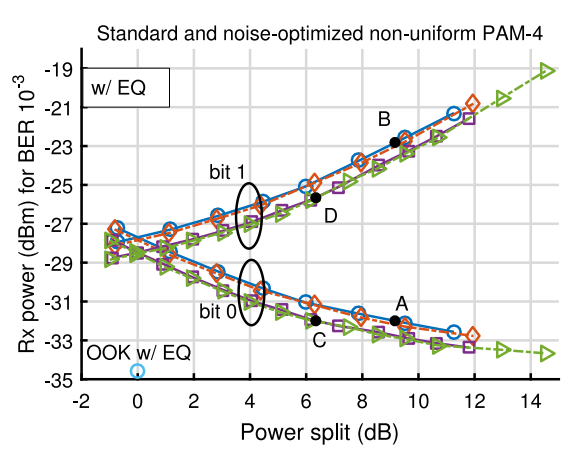

(b)

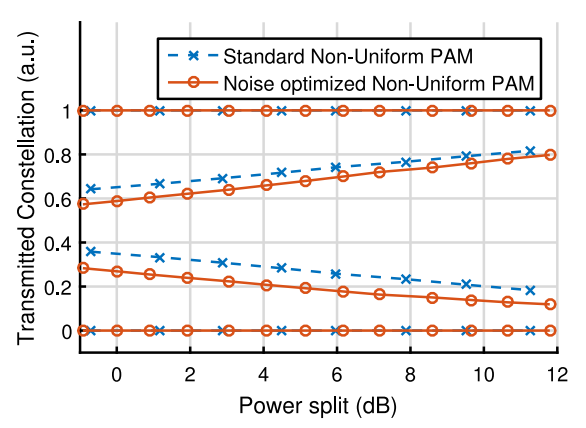

(c)

Fig. 7. (a,b): Experimental results showing the required received optical power at the SOA-PIN receiver to decode a bit at a BER level of $10^{-3}$ for standard non-uniform PAM signals and for signal-dependent noise optimized non-uniform PAM signals. The symbol rate is constant at 10 Gbaud. B2B and 40 km results are shown as solid, and dashed lines, respectively. (a): Shows performance without the use of an equalizer (b): Shows performance with a 31 tap FFE. (c): Normalized transmitted constellation for the standard non-uniform PAM and signal-dependent noise optimized non-uniform PAM as a function of the measured power split for B2B transmission with FFE.

TABLE I

PERCENTAGE OF ONUS IN THE INEA NETWORK THAT CAN DECODE VARIOUS FORMS OF PAM BASED ON THE PRESENTED MEASUREMENTS

\begin{tabular}{|c|c|c|}
\hline APD-BASED RECEIVER & w/o FFE & w/ FFE \\
\hline \multicolumn{3}{|l|}{ Without non-uniform PAM } \\
\hline Equidistant PAM-4 & $64 \%$ & $77 \%$ \\
\hline Equidistant PAM-8 & - & $5 \%$ \\
\hline \multicolumn{3}{|l|}{ With non-uniform PAM } \\
\hline Standard non-uniform PAM-4 & $75 \%$ & $87 \%$ \\
\hline Standard non-uniform PAM-8 & - & $8 \%$ \\
\hline SOA-PIN BASED RECEIVER & w/o FFE & w/ FFE \\
\hline \multicolumn{3}{|l|}{ Without non-uniform PAM } \\
\hline Equidistant PAM-4 & $54 \%$ & $61 \%$ \\
\hline Noise-dependent PAM-4 & $64 \%$ & $71 \%$ \\
\hline \multicolumn{3}{|l|}{ With non-uniform PAM } \\
\hline Standard non-uniform PAM-4 & $64 \%$ & $71 \%$ \\
\hline Noise-dependent non-uniform PAM-4 & $75 \%$ & $82 \%$ \\
\hline
\end{tabular}

improvement for OOK. This increased power penalty due to SOA-PIN receiver can, for a large part, be prevented by using a signal-dependent noise optimized constellation.

An APD receiver also has a signal-dependent noise contribution, although with a relatively lower contribution in comparison to the SOA-PIN receiver. In an experiment with the presented algorithm, we observed that the increase of the number of users equipped with non-uniform PAM is $1 \%$. This is a negligible increase, hence not considered further in this paper.

\section{CONClusions}

Non-uniform PAM uses an unequal distribution of the amplitude levels. Therefore, decoding of some bits in the PAM symbol is possible at a lower received optical power level, while other bits require a higher received optical power level. This can be utilized in practical PON deployments where there is a spread in received optical powers among the ONUs. Spreads of more than $15 \mathrm{~dB}$ have been found. In a PON with flexible modulation and non-uniform PAM, the obtainable increase in aggregated data rate depends on the distribution of users at a certain OLT port. Based on our experimental results with an APD-based receiver, and the examined loss statistics of a deployed PON, the aggregated data rate increases from $10 \mathrm{Gbps}$ to $18.7 \mathrm{Gbps}$ with the use of flexible non-uniform PAM-4. Additional support for non-uniform PAM-8 increases the aggregated data rate somewhat further to 19.4 Gbps due to the high additional penalty of PAM- 8 relative to the power range that is applicable for PONs. In addition to a flexible modulation scheme, non-uniform PAM can help to combat component ageing to a certain degree, e.g. if a certain ONU's performance decreases over time, this can be counteracted by pairing this ONU to another ONU that yet has to show ageing effects, and that still performs better than required.

In systems where signal-dependent noise contributes significantly to the total noise level, optimization of the transmitted constellation can improve the performance. At the ONUs, the received signals will then be evaluated at different received optical power levels, each with their own relative signaldependent noise level. For systems where signal-dependent noise plays a dominant role, based on the experimental work and the loss statistics of the deployed PON, the aggregated capacity of a PON can be increased by an additional $11 \%$ if this optimization is performed.

\section{ACKNOWLEDGements}

This work is made possible by INEA for the GPON statistics and by the Netherlands Organisation for Scientific Research (NWO) through grant 13530.

\section{REFERENCES}

[1] L. Xue, L. Yi, H. Ji, P. Li, and W. Hu, "First demonstration of symmetric 100G-PON in O-band with 10G-class optical devices enabled by dispersion-supported equalization," in 2017 Optical Fiber Communications Conference and Exhibition (OFC), Mar. 2017, pp. 1-3. 
[2] ITU-T, "G.9807.1 : 10-Gigabit-capable symmetric passive optical network (XGS-PON)," 2016

[3] R. van der Linden, N. C. Tran, E. Tangdiongga, and A. M. J. Koonen, "Increasing flexibility and capacity in real PON deployments by using 2/4/8-PAM formats," IEEE/OSA Journal of Optical Communications and Networking, vol. 9, no. 1, pp. A1-A8, Jan. 2017.

[4] T. Cover, "Broadcast channels," IEEE Transactions on Information Theory, vol. 18, no. 1, pp. 2-14, Jan. 1972.

[5] K. Ramchandran, A. Ortega, K. M. Uz, and M. Vetterli, "Multiresolution broadcast for digital HDTV using joint source/channel coding," IEEE Journal on Selected Areas in Communications, vol. 11, no. 1, pp. 6-23, Jan. 1993.

[6] U. Reimers, "DVB-T: The COFDM-based system for terrestrial television," Electronics Communication Engineering Journal, vol. 9, no. 1, pp. 28-32, Feb. 1997.

[7] R. van der Linden, N.C. Tran, E. Tangdiongga, and A.M.J. Koonen, "Improvement on Received Optical Power Based Flexible Modulation in a PON by the Use of Non-Uniform PAM," in ECOC 2017; 43th European Conference on Optical Communication, Sep. 2017.

[8] M. D. Santa, C. Antony, G. Talli, and P. D. Townsend, "Power Budget Improvement in Passive Optical Networks Using PAM4 Hierarchical Modulation," IEEE Photonics Technology Letters, vol. 29, no. 20, pp. 1747-1750, Oct. 2017.

[9] V. Houtsma, D. van Veen, and H. Chow, "Demonstration of Symmetrical $25 \mathrm{~Gb} / \mathrm{s}$ TDM-PON With Multilevel Interleaving of Users," Journal of Lightwave Technology, vol. 34, no. 8, pp. 2005-2010, Apr. 2016.

[10] Y. Li, H. Dai, G. Shen, and S. K. Bose, "Adaptive FEC selection for lightpaths in elastic optical networks," in Optical Fiber Communications Conference and Exhibition (OFC), 2014, Mar. 2014, pp. 1-3.

[11] Wei Yang, "Experimental results of SOA pre-amplification for 25GEPON,” IEEE P802.3ca Task Force Meeting, Mar. 2017, iEEE P802.3ca Task Force Meeting, yang 3ca_1a 0317.

[12] G. P. Agrawal, Lightwave Technology: Telecommunication Systems. John Wiley \& Sons, Aug. 2005.

[13] K. Ho and J. M. Kahn, "Multilevel optical signals optimized for systems having signal-dependent and signal-independent noises, finite transmitter extinction ratio and intersymbol interference," U.S. Patent US6 690894 B2, Feb., 2004.

Robbert van der Linden (S'08) Robbert van der Linden studied Electrical Engineering at Eindhoven University of Technology, Eindhoven, the Netherlands. In 2013, he received his M.Sc. degree (cum laude) from Eindhoven University of Technology. Afterwards, he joined the COBRA Research Institute (now named Institute for Photonic Integration (IPI)), working toward a PhD degree on flexible access networks in close collaboration with Genexis B.V. His research interests include optical access networks and flexible networks.
Eduward Tangdiongga (S'01, M'10) received the M.Sc. and Ph.D. degrees from the Eindhoven University of Technology, Eindhoven, The Netherlands, in 1994 and 2001, respectively. In 1994, he joined the COBRA Research Institute (currently named Institute for Photonic Integration), working on high-speed optical switches. From 2001 he has participated in several European Union and Dutch sponsored research programs targeting for integration of various wired and wireless services on a single optical in-building network. In 2006, he was appointed as an Assistant Professor on short-haul optical communication. His research interest includes random signals, ultrafast dynamics in semiconductor optical devices, adaptive access networks, radio over (single mode-, multimode-, and plastic) fiber, and optical wireless.
Ton Koonen (IEEE F'07, OSA F'13) is full professor in Eindhoven University of Technology since 2001. Since 2004 he is chairman of the group Electro-Optical Communication Systems and since 2012 vice-dean of the department Electrical Engineering. Before 2001, he worked for more than 20 years in applied research in industry, amongst others in Bell Labs - Lucent Technologies. He is Bell Labs Fellow (1998), IEEE Fellow (2007), OSA Fellow (2013), and Distinguished Guest Professor of Hunan University, Changsha, China (2014). In 2011 he received an Advanced Investigator Grant of the European Research Council. His current research interests are spatial division multiplexed systems, access and in-building fiber networks, including high-capacity POF networks, radio-over-fiber techniques, and wireless optics techniques.
Nguyen-Cac Tran (S'06) received the B.Eng. degree in electronics and telecommunications from the Hanoi University of Science and Technology, Hanoi, Vietnam, in 2005, the M.Sc.Eng. degree in Electrical Engineering from Korea University, in February 2008, and Ph.D. degree in optical communications from COBRA Research Institute, Eindhoven University of Technology, The Netherlands, in March 2013. From August 2005 to March 2006, he was a Junior Network Administrator at Vietnam Telecom National. In 2008, he joined Panasonic Hanoi Laboratory as a scientific assistant for the director. Since 2013, he has been with Genexis B.V. as product line manager for FTTH residential gateway equipment. His research interests include optical fiber access and in-building networks, optical network control and management, and deploying solutions for fiber to/in the home. 